\title{
Erratum: Influence of a Structural Transition on the Optical and the Magneto-optical Properties of $\mathrm{Co}_{2} \mathrm{MnGa}$ Alloy Films
}

\author{
[J. Korean Phys. Soc. 49, 2180 (2006)] \\ K. W. KIM* \\ Department of Physics, Sunmoon University, Asan 336-708 \\ R. J. KIM and Y. P. LEE \\ Quantum Photonic Science Research Center and BK21 Program Division of \\ Advanced Research and Education in Physics, Hanyang University, Seoul 133-791 \\ J. Y. RHEE \\ BK21 Physics Research Division and Institute of Basic Science, Sungkyunkwan University, Suwon 440-746 \\ Y. V. KuDRYAVISEV \\ Institute of Metal Physics, National Academy of Sciences of Ukraine, 36 Vernadsky str, 03142, Kiev-142, Ukraine
}

DOI: $10.3938 /$ jkps.62.1709

The correct name of the last Author is 'Y. V. Kudryavtsev'. 